\title{
Entanglement of separate nitrogen-vacancy centers coupled to a whispering-gallery mode cavity
}

\author{
W. L. Yang, ${ }^{1}$ Z. Y. Xu, ${ }^{1,2}$ M. Feng, , 田 and J. F. Du, 团 \\ ${ }^{1}$ State Key Laboratory of Magnetic Resonance and Atomic and Molecular Physics, \\ Wuhan Institute of Physics and Mathematics, Chinese Academy of Sciences, Wuhan 430071, China \\ ${ }^{2}$ Graduate School of the Chinese Academy of Sciences, Beijing 100049, China \\ ${ }^{3}$ Hefei National Laboratory for Physics Sciences at Microscale and Department of Modern Physics, \\ University of Science and Technology of China, Hefei, 230026, China
}

\begin{abstract}
We present a quantum electrodynamical model involving nitrogen-vacancy centers coupled to a whispering-gallery mode cavity. Two schemes are considered to create W state and Bell state, respectively. One of the schemes makes use of Raman transition with the cavity field virtually excited and the other enables Bell state preparation and quantum information transfer by virtue of dark state evolution and adiabatic passage, which is tolerant to ambient noise and experimental parameter fluctuations. We justify our schemes by considering the experimental feasibility and challenge using currently available technology.
\end{abstract}

PACS numbers:

\section{INTRODUCTION}

The diamond nitrogen-vacancy (NV) center consisting of a substitutional nitrogen atom and an adjacent vacancy has attracted considerable attention since the first report of optically detected magnetic resonance on single NV center in 1997 [1]. Due to sufficiently long electronic spin lifetime as well as the possibility of coherent manipulation at room temperature [2], the NV center is considered as a promising building block for room-temperature quantum computing in the future [3-5].

Since the electronic spins could be well initialized and manipulated in optical fashion, the qubit readout and gating regarding single-spin state have been achieved in individual NV centers [6]. By virtue of the hyperfine couplings with the paramagnetic nuclei in the vicinity of the electron spin, i.e., ${ }^{13} \mathrm{C}$ [7], ${ }^{14} \mathrm{~N}$ [8], and ${ }^{15} \mathrm{~N}$ [9], currently available techniques have demonstrated the quantum information storage and retrieval between electronic and the nuclear spins [7]. This technique also enables rapid and high-fidelity readout of quantum information from the electron spin [10]. However, coherence between electron and nuclear spin qubits is restricted to the case of a few qubits owing to the limited number of nuclear spins individually addressable in frequency space 11, 12]. So for scalability, it is necessary to develop methods of coupling distant NV centers.

We have noticed a recent experiment to entangle a pair of separate NV centers within a diamond based on magnetic dipolar coupling [13]. But this idea is pretty hard for scalability. For distant NV centers with magnetic dipolar coupling unavailable, the best way for spin-spin entanglement seems to make use of parity projection by detecting the emitted photons relevant to different spin

\footnotetext{
*mangfeng@wipm.ac.cn

${ }^{\dagger}$ djf@ustc.edu.cn
}

states 14. However, the NV centers, although similar to the atomic cases, only allow linearly polarized radiations in the laser excitation, which makes coincident detection of emitted photons unavailable. In addition, to effectively produce entanglement of NV centers by parity projection of photons, we require that the 'which-path' information be removed due to interference after the photons go through the beam splitter. But experimental reports so far have shown that $96 \%$ of the emitted photons reside in broad photon sidebands to the resonant zero phonon line (ZPL) at $637 \mathrm{~nm}$ even in cryogenic situation 14]. This implies that the most photons emitted from the NV centers could not effectively interfere in the beam splitter.

Alternatively, the entanglement of separate NV centers could be achieved by coupling to the same cavity mode. In a recent publication [15], we proposed an idea to entangle more than two NV centers by one step of implementation, based on coupling to a microsphere cavity. The key idea of that proposal is the employment of the spin singlet state ${ }^{1} A$ to encode a qubit. To our knowledge, however, this metastable ${ }^{1} A$ state, although investigated from $C_{3 v}$ group theory considerations [16] and other aspects, has not yet been fully understood [17]. As a result, most of the present work for quantum information processing with NV centers encode qubits only in the sublevels of the ground state ${ }^{3} A$.

We focus in this work on entangling distant NV centers without employing the state ${ }^{1} A$. Specifically, we encode qubits in two of the ground state splittings, and the excited states are auxiliary with spontaneous emission effectively suppressed during our operation. The key point of our idea is to present a generalized Jaynes-Cummings model involving a quantized whispering-gallery mode (WGM) and $N$ identical NV centers. WGM microcavities are of typically dielectric rotational-symmetry structures with WGMs traveling around the curved boundary and confined by continuous total internal reflection [18]. In particular, the technological advance has made 
it available to have strong light-matter coherent coupling in WGM resonators with smaller mode volume $V_{m}$ and extremely high-quality factor $Q[19,20]$. Recent experimental progresses about the nanocrystal-microsphere system also provide experimental evidence for strong coupling between NV centers and the WGM of silica microsphere [21], polystyrene microsphere [22], and galliumphosphide microdisk [23], respectively. In addition, a latest experiment has demonstrated the technique for deterministically coupling a single NV center to a photonic crystal cavity [24]. So far there have been much development in WGM cavities with the forms, such as the microtoroidal [25], microcylinders [26], microdisks [27], and microspheres 28].

We will show how to generate $W$ state [29] and Bell states for these distant NV centers in such a composite nanocrystal-microsphere system. The main results of this work are twofold: First, by virtue of Raman transitions, we show the possibility with virtual-photon-induced excitation in a large detuning case to generate multipartite W state with separate NV centers in different diamond nanocrystals, where the growth of the qubit number corresponds to the decrease of the operational time. Secondly, resorting to adiabatic passage technology [30, 31], we create Bell states of any pairs of qubits via dark-state evolution, which is robust to the cavity decay.

\section{ENTANGLEMENT GENERATION BY RAMAN CONFIGURATION}

\section{A. Effective Hamiltonian}

As sketched in Fig. 1(a) and 1(b), $N$ identical NV centers, respectively, in $N$ separate diamond nanocrystals could be strongly coupled to the WGM of a microsphere cavity or of a microtoroidal cavity. The NV center is a point defect in the diamond lattice, which consists of a nearest-neighbor pair of a nitrogen-atom impurity substituting a carbon atom and a adjacent carbon vacancy, as shown in Fig. 1(c). In the case of microspheres, WGM can be characterized by angular $l$, azimuthal $m$, and radial $s$ numbers. High values of $Q$ usually correspond to the modes with $l \gg 1$. Of greatest interest is the socalled fundamental WGM $(s=1, l=m)$, whose field is concentrated in the vicinity of the equatorial plane of the sphere. It is believed that these modes can be selectively excited by coupling to a tapered fiber [32]. Like in [15], our proposal is also based on recent experimental and theoretical progresses, i.e., the possibility of $\Lambda$-type configuration of the optical transition in NV center system 33] and the considerable enhancement of the ZPL by embedding the NV centers in some cavities [34]. But differently, the entanglement of the NV centers is achieved without involving the metastable ${ }^{1} A$ state. In our case, we assume that each NV center located in a diamond nanocrystal is attached around the equator of a single fused-silica microsphere cavity [28] or microtoroidal cav- (a)

(b)

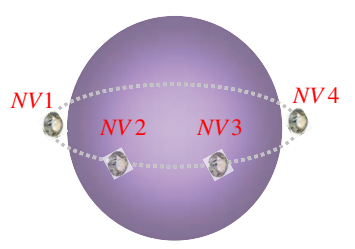

Microsphere Cavity

(c)

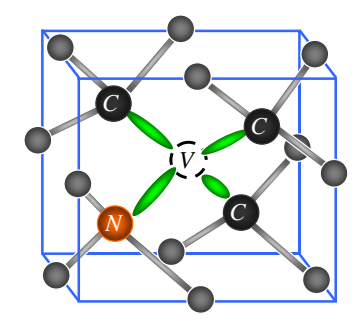

(d)

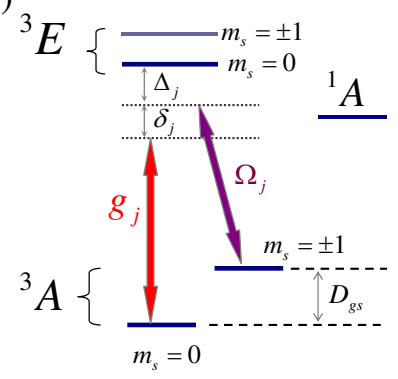

FIG. 1: (a) Schematic setup in the microsphere cavity case, where $N$ identical NV centers in diamond nanocrystals are equidistantly attached around the equator of a single fusedsilica microsphere cavity. (b) Schematic setup in the microtoroidal cavity case, where $N$ identical NV centers in the vicinity of the microtoroidal cavity are able to interact with the WGM via the evanescent field. (c) Structure of the NV color center in the diamond lattice, consisting of a substitutional nitrogen $(\mathrm{N})$ and a neighboring vacancy $(\mathrm{V})$. (d) Level diagram for the $j$-th NV center, where $\Delta_{j}$ and $\delta_{j}$ are detunings, $g_{j}$ is the coupling strength between NV center and WGM, and $\Omega_{j}$ is the coupling strength between NV center and the laser pulse. $D_{g s}=2.88 \mathrm{GHz}$ is the zero-field splitting between the ground state sublevels $m_{s}=0$ and $m_{s}= \pm 1$ $\left(m_{s}= \pm 1\right.$ are degenerate at zero magnetic field due to $C_{3 v}$ symmetry) of the NV center. We encode qubits in the subspace spanned by the down state $m_{s}=0$ and the up state $m_{s}=-1$.

ity [25]. This composite nanocrystal-microsphere system takes advantage of the exceptional spin properties of $\mathrm{NV}$ centers as well as the ultrahigh quality factor $Q\left(\geq 10^{8}\right.$ even up to $\left.10^{10}\right)$, very small volume $\left(V_{m} \leq 100 \mu m^{3}\right)$ and simple fabrication technique of the cavity 35]. For convenience of description, we will below mention the WGM, but not relating the mode to any concrete cavity.

By combining laser pulses with carefully timed interaction with the WGM, one can model the NV center as a $\Lambda$-type three-level system as shown in Fig. 1(d), where the states $\left|{ }^{3} A, m_{s}=0\right\rangle$ and $\left|{ }^{3} A, m_{s}=-1\right\rangle$ serve as the logical states $|0\rangle$ and $|1\rangle$ of the qubit, respectively, and the state $\left|{ }^{3} E, m_{s}=0\right\rangle$ is labeled by the state $|e\rangle$. In our case, the WGM with frequency $\omega_{c}$ is far-off resonant from the transition $|0\rangle \Longleftrightarrow|e\rangle$ (with the frequency $\omega_{e 0}$ ), and the levels $|1\rangle$ and $|e\rangle$ (with transition frequency $\omega_{e 1}$ ) are coupled by a largely detuned laser with frequency $\omega_{L}$ and polarization $\sigma^{+}[33,[36]$. The NV centers are fixed 
and apart with the distance much larger than the wavelength of the WGM, interacting individually with laser beams. So the direct coupling between NV centers is negligible. Assuming that the detuning $\Delta_{j}$ is sufficiently larger than the coupling strength $g_{j}$ and $\Omega_{j}$, the excited state $|e\rangle$ can be adiabatically eliminated. Thus quantum logic gates and multipartite entangled states are available in the subspace spanned by $|0\rangle$ and $|1\rangle$. Using the rotating-wave approximation (RWA), the Hamiltonian in the interaction picture can be written in units of $\hbar=1$ as 37.

$$
H_{I}=\sum_{j=1}^{N} \eta_{j}\left[a^{+} \sigma_{j}^{-} e^{-i \delta_{j} t}+a \sigma_{j}^{+} e^{i \delta_{j} t}\right],
$$

where $\sigma_{j}^{+}=\left|1_{j}\right\rangle\left\langle 0_{j}\left|, \sigma_{j}^{-}=\right| 0_{j}\right\rangle\left\langle 1_{j}\right|$, and $a^{+}(a)$ is the creation (annihilation) operator of the WGM field. $\eta_{j}=$ $g_{j} \Omega_{j}\left(\frac{1}{\Delta_{j}+\delta_{j}}+\frac{1}{\Delta_{j}}\right)$ with $\Delta_{j}=\omega_{e 1, j}-\omega_{L, j}$ and $\delta_{j}=\omega_{e 0, j}+$ $\omega_{L, j}-\omega_{e 1, j}$. For simplicity, we assume that the detuning $\delta_{j}$ and the interaction term $\eta_{j}$ are identical for each qubit, that is, $\eta_{j}=\eta$, and $\delta_{j}=\delta$. In the case of $\delta \gg \eta$, there is no energy exchange between the WGM and NV centers. If the quantized WGM is initially in the vacuum state, the effective Hamiltonian could be simplified as 38]

$$
H_{\text {eff }}=\gamma\left[\sum_{j=1}^{N}\left|1_{j}\right\rangle\left\langle 1_{j}\right|+\sum_{j, k=1, j \neq k}^{N} \sigma_{j}^{+} \sigma_{k}^{-}\right]
$$

where the first term corresponds to the dynamical energy shift regarding the level $|1\rangle$, and the photon-dependent energy shift of the level $|0\rangle$ is removed due to the vacuum state of the cavity. The rest terms in Eq. (2) denote the coupling between any pair of NV centers through the WGM, and $\gamma=\left|n^{2} / \delta\right|$ is the effective coupling strength

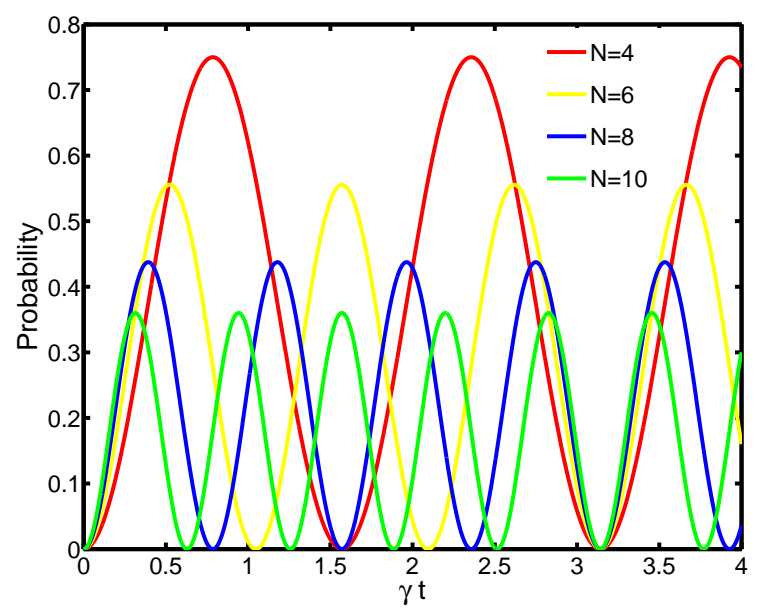

FIG. 2: The probability of obtaining the state $|W\rangle_{N-1}$ versus $\gamma t$.

\section{B. Creation of $\mathrm{W}$ state}

Let us first consider the creation of $N$-qubit $W$ state for NV centers. If the first $(N-1) \mathrm{NV}$ centers are initially prepared in the state $|00 \cdots 0\rangle_{1,2, \cdots, N-1}$ and only the $N$ th NV center is initially prepared in state $|1\rangle_{N}$, one can easily get the following time-dependent state evolution,

$$
|\Psi(t)\rangle=C_{1}|00 \cdots 0\rangle_{1,2, \cdots, N-1}|1\rangle_{N}+C_{2}|W\rangle_{N-1}|0\rangle_{N},
$$

with the coefficients $C_{1}=\left(e^{-i N \gamma t}+N-1\right) / N$ and $C_{2}=\sqrt{N-1}\left(e^{-i N \gamma t}-1\right) / N .|W\rangle_{N-1}=(1 / \sqrt{N-1})$ $|N-2,1\rangle$ is the generalized form of the $W$ state, which denotes the symmetric state involving $(N-2)$ zeroes and 1 one. According to Eq. (3), the state of other $(N-1)$ NV centers will surely collapse into the state $|W\rangle_{N-1}$ in the case of the measurement on the $N$-th NV center being $|0\rangle_{N}$. As a result, we can find that the probability of obtaining the state $|W\rangle_{N-1}$ is $\left|C_{2}\right|^{2} /\left(\left|C_{1}\right|^{2}+\left|C_{2}\right|^{2}\right)$, which gets to maximum $P_{\max }=4(N-1) /\left[4(N-1)+(N-2)^{2}\right]$ at $t_{N}=(2 k+1) \pi / N \gamma$ with $k$ non-negative integers.

Fig. 2 shows that the gating time $t_{N}$ is inversely proportional to the qubit number $N$, so is the maximal probability $P_{\max }$. In this composite nanocrystal-microsphere system, the coupling between the NV center and WGM could reach $g_{\max }=\Gamma_{0}\left|\vec{E}(r) / \vec{E}_{\max }\right| \sqrt{V_{a} / V_{m}}[20]$, where $\left|\vec{E}(r) / \vec{E}_{\max }\right|$ is the normalized electric field strength at the location $r$, and $V_{a}=3 c \lambda^{2} / 4 \pi \Gamma_{0}$ denotes a characteristic interaction volume with $\lambda$ the transition wavelength between the states $|e\rangle$ and $|0\rangle, \Gamma_{0}$ the spontaneous decay rate of the excited state $|e\rangle$ and $c$ the speed of light. Using the values $\lambda=637 \mathrm{~nm}, \Gamma_{0}=2 \pi \times 83 \mathrm{MHz}$ [39], $V_{m}=100 \mu m^{3}$, and $\left|\vec{E}(r) / \vec{E}_{\max }\right|=1 / 6$, we have the $\max -$ imal coupling $g_{\max } \simeq 2 \pi \times 1 \mathrm{GHz}$, and the other experimental parameters can be adjusted as $\Omega_{j}=2 \pi \times 100$ $\mathrm{MHz}, \Delta_{j}=2 \pi \times 10 \mathrm{GHz}$, and $\delta_{j}=2 \pi \times 100 \mathrm{MHz}$. Provided $\eta_{j}=2 \pi \times 20 \mathrm{MHz}$, we have $\gamma=\left|\eta^{2} / \delta\right|=2 \pi \times 4$ $\mathrm{MHz}$, and the operation time $t_{N}$ to be $0.0313 \mu \mathrm{s}, 0.0208$ $\mu \mathrm{s}$, and $0.0156 \mu \mathrm{s}$ in the case of $N=4, N=6$, and $N=8$, respectively.

\section{Estimate of decoherence}

We now consider the influence due to decoherence, which results from the effective spontaneous emission from the states $|1\rangle$ to $|0\rangle$. Here we have neglected the WGM decay because the cavity decay rate could be $\kappa=\omega_{e 0} / Q=2 \pi \times 0.47 \mathrm{MHz}$ in the case of $Q=10^{9}$, which is much smaller than the effective coupling rate $\gamma$. The characteristic spontaneous emission rate $\Gamma_{\text {eff }}$ regarding the states $|1\rangle$ and $|0\rangle$ could be estimated as $\Gamma_{0} \Omega_{j} g_{j} / \Delta_{j}^{2}$ [40], where $\Gamma_{0}$ is the spontaneous decay rate of the excited state $|e\rangle$ [41]. So the evolution of the system is described by the Lindblad equation [42]

$$
\dot{\rho}=-i[H, \rho]+\Gamma_{e f f}\left(2 \sigma^{-} \rho \sigma^{+}-\sigma^{+} \sigma^{-} \rho-\rho \sigma^{+} \sigma^{-}\right) .
$$

Fig. 3 shows the fidelity of the state $|W\rangle_{4}$ when the spontaneous decay is considered. With increase of $\Gamma_{e f f}$, the fidelity decreases accordingly. However, our scheme 

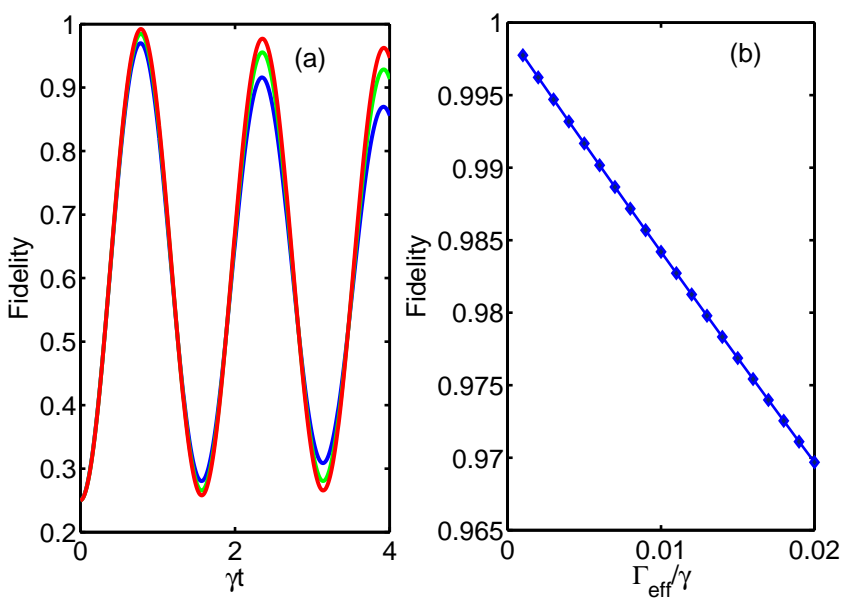

FIG. 3: (a) The fidelity of the state $|W\rangle_{4}$ versus $\gamma t$, where the blue, green and red curves correspond to $\Gamma_{\text {eff }}=\gamma / 50$, $\gamma / 100$, and $\gamma / 200$, respectively. (b) The fidelity of the state $|W\rangle_{4}$ versus $\Gamma_{\text {eff }} / \gamma$, where the gating time is $\pi / 4 \gamma$.

can still achieve a high fidelity as long as the spontaneous decay is weak. In a realistic experiment, the situation would be more complicated than our consideration above. As a result, to carry out our scheme with high efficiency and high fidelity, we have to suppress above mentioned imperfect factors as much as we can.

\section{ENTANGLEMENT GENERATION BY ADIABATIC PASSAGE OF DARK STATES}

\section{A. Dark State Evolution}

Under Raman resonance conditions between two Zeeman sublevels of the ground state, we focus on generating Bell state with any pairs of NV centers (e.g., $A$ and $B$ ) in the nanocrystal-microsphere system, via adiabatic passage of the dark states [43]. The adiabatic passage [44] is a useful and robust technique for quantum-state manipulation. It is a method of using two time-separated but partially overlapping pulses in the counterintuitive sequence to produce complete population transfer or an arbitrary coherent superposition between the initial and final states.

The difference from the above section is that the WGM and the laser pulses should be in resonance with the transitions $|0\rangle \Longleftrightarrow|e\rangle$ and $|1\rangle \Longleftrightarrow|e\rangle$, respectively, as shown in Fig. 4.

The interacting Hamiltonian, after RWA, has the form

$$
H_{I}^{\prime}=\sum_{j=A, B}\left[g_{j} a^{+}\left|0_{j}\right\rangle\left\langle e_{j}\left|+\Omega_{j}(t)\right| e_{j}\right\rangle\left\langle 1_{j}\right|+H . c .\right],
$$

by which the Bell state is generated following the procedure below: (i) The NV centers $A$ and $B$ are prepared in the initial state $\left|1_{A}\right\rangle\left|0_{B}\right\rangle$, and the WGM is initially prepared in vacuum state $\left|0_{c}\right\rangle$; (ii) We set initially the Rabi

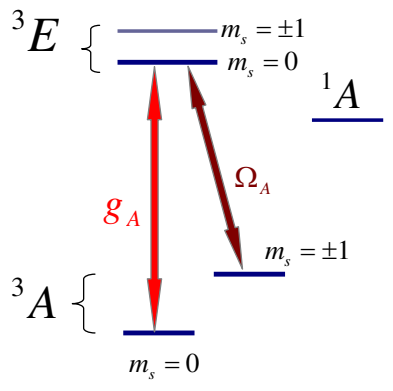

NV Center $A$

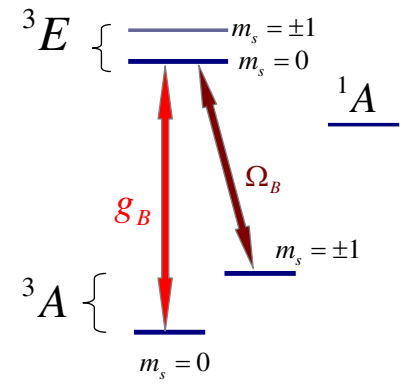

NV Center $B$
FIG. 4: Level diagram for two NV centers $A$ and $B$, where $g_{j}(j=A$ and $B)$ is the coupling constant due to the WGM, and $\Omega_{j}(t)$ is the Rabi frequencies relevant to laser pulses.

frequencies $\Omega_{A} \ll \Omega_{B}$, and then we adiabatically decrease $\Omega_{B}$ while increasing $\Omega_{A}$, driving the system into a superposition of Zeeman sublevels which by quantum interference are decoupled from the excited state and form a dark state $|D\rangle$ (defined later); (iii) We go on adiabatically decreasing $\Omega_{B}$ while increasing $\Omega_{A}$ until $\Omega_{A}=$ $\Omega_{B}=\Omega_{0}$, we reach the state $\left|D_{f}\right\rangle$ and then turn off the laser pulses. These steps can be briefly described as

$$
\left.\left|1_{A}\right\rangle\left|0_{B}\right\rangle\left|0_{c}\right\rangle \stackrel{(\mathrm{ii})}{\longrightarrow}|| D\right\rangle(\mathrm{iii})\left|D_{f}\right\rangle,
$$

where

$$
\begin{aligned}
& |D\rangle=\tilde{N}^{\prime}\left[\Omega_{B}(t) g_{A}\left|1_{A}\right\rangle\left|0_{B}\right\rangle\left|0_{c}\right\rangle+\Omega_{A}(t) g_{B}\left|0_{A}\right\rangle\left|1_{B}\right\rangle\left|0_{c}\right\rangle\right. \\
& \left.-\Omega_{A}(t) \Omega_{B}(t)\left|0_{A}\right\rangle\left|0_{B}\right\rangle\left|1_{c}\right\rangle\right]
\end{aligned}
$$

is a dark state regarding the Hamiltonian in Eq. (5), and

$$
\begin{aligned}
& \left|D_{f}\right\rangle=\tilde{N}\left[\Omega_{0}(t)\left(g_{A}\left|1_{A}\right\rangle\left|0_{B}\right\rangle\left|0_{c}\right\rangle+g_{B}\left|0_{A}\right\rangle\left|1_{B}\right\rangle\left|0_{c}\right\rangle\right)\right. \\
& \left.-\Omega_{0}^{2}(t)\left|0_{A}\right\rangle\left|0_{B}\right\rangle\left|1_{c}\right\rangle\right]
\end{aligned}
$$

is the final state with $\tilde{N}$ and $\tilde{N}^{\prime}$ being normalization factors. In the case of $g_{A}=g_{B}=g_{0}$, the final state $\left|D_{f}\right\rangle$ is simplified to

$$
\left|D_{f}\right\rangle=\tilde{N}\left[\sqrt{2} \Omega_{0}(t) g_{0}|B e l l\rangle_{A B}\left|0_{c}\right\rangle-\Omega_{0}^{2}(t)\left|0_{A}\right\rangle\left|0_{B}\right\rangle\left|1_{c}\right\rangle\right]
$$

with the Bell state $\mid$ Bell $\rangle_{A B}=\left(\left|1_{A}\right\rangle\left|0_{B}\right\rangle+\left|0_{A}\right\rangle\left|1_{B}\right\rangle\right) / \sqrt{2}$. Our interest is in the components of the vacuum state $\left|0_{c}\right\rangle$ of the WGM, which as shown in Eq. (9) correspond to the Bell state, and we have to project the WGM on the state $\left|0_{c}\right\rangle$ [45]. To obtain the Bell state $|B e l l\rangle$ with high success rates, we need to ensure that the coupling strength $g_{0}$ is much larger than the Rabi frequency of the laser pulse $\Omega_{0}$. So noteworthy features of our scheme are as follows: (i) In principle, the excited states of NV centers are negligibly populated due to dark state dominant in the evolution, so the preparation of the Bell state is immune to spontaneous emission; (ii) The cavity decay exists only for a short time with the intermediate state $\left|1_{c}\right\rangle$ populated, whereas the satisfied condition $g_{0} \gg \Omega_{0}$ 
makes the detrimental influence from the cavity decay negligible during the operations. We will justify these points by numerics later.

Note that the method of dark-state evolution can be also applied to quantum information transfer (QIT) between any pairs of NV centers, where the the original quantum information is encoded on the NV center $A$ as $\left|\Psi_{0}\right\rangle=c_{0}\left|0_{A}\right\rangle+c_{1}\left|1_{A}\right\rangle\left(c_{0}\right.$ and $c_{1}$ are arbitrary coefficients), which can be coherently transferred to the NV center $B$ (initially prepared in $\left|0_{B}\right\rangle$ state) via Raman transitions induced by a pair of time-delayed laser pulses. We can apply such a "counterintuitive" pulse sequence from $\Omega_{A}(t) / \Omega_{B}(t) \ll 1$ to $\Omega_{A}(t) / \Omega_{B}(t) \gg 1$, namely, the pulse on NV center $B$ precedes the pulse on NV center $A$, which guarantees that the adiabatic transfer of the quantum information could be achieved. Compared with the creation of Bell state, there is only a slight modification on the step (iii) in above mentioned procedure, i.e., slowly changing the Rabi frequencies to meet the condition $\Omega_{A} \gg \Omega_{B}$, rather than $\Omega_{A}=\Omega_{B}$. In addition, different from in preparing Bell state, the initial state $\left|\Psi_{0}\right\rangle\left|0_{B}\right\rangle\left|0_{c}\right\rangle$ will drive the system to undergo a different dark-state evolution involving two dark states $|D\rangle$ and $|D\rangle^{\prime}$, where $|D\rangle^{\prime}=\left|0_{A}\right\rangle\left|0_{B}\right\rangle\left|0_{c}\right\rangle$ is another dark state regarding the Hamiltonian in Eq. (5). The QIT process could be briefly expressed as

$$
\begin{aligned}
& \left(c_{0}\left|0_{A}\right\rangle+c_{1}\left|1_{A}\right\rangle\right)\left|0_{B}\right\rangle\left|0_{c}\right\rangle \underset{(\mathrm{ii})}{\stackrel{(\mathrm{ii})}{\longrightarrow}}\left|0_{0}\right\rangle 0_{A}|D\rangle^{\prime}+c_{1}|D\rangle \\
& \stackrel{\left.\left.0_{B}\right\rangle+c_{1}\left|1_{B}\right\rangle\right)\left|0_{c}\right\rangle .}{\longrightarrow}
\end{aligned}
$$

\section{B. Decay Case}

Without losing generality, we consider two NV centers with the identical parameters and two laser pulses with Gaussian envelops $\Omega_{A}(t)=\Omega_{m} e^{-\left(t-\tau_{A}\right)^{2} / \triangle \tau^{2}}$ and $\Omega_{B}(t)=\Omega_{m} e^{-\left(t-\tau_{B}\right)^{2} / \triangle \tau^{2}}$, where $\Omega_{m}$ is the maximal value of $\Omega_{(A) B}$ at the central time $\tau_{j}$ for the pulse $j$ $(j=A, B), \triangle \tau$ is the laser beam waist. Fig. 5 presents the numerical treatment for the QIT process: $\left|1_{A}\right\rangle\left|0_{B}\right\rangle\left|0_{C}\right\rangle \rightarrow\left|0_{A}\right\rangle\left|1_{B}\right\rangle\left|0_{c}\right\rangle$. We have compared the population of $\left|0_{A}\right\rangle\left|1_{B}\right\rangle\left|0_{C}\right\rangle$ in an ideal dark-state evolution described by Eq. (5) with that in the decay case. If no photon leakage really happens either from the excited state or from the cavity mode during the gating period, the system is governed by

$$
\begin{aligned}
H_{\text {decay }}= & \sum_{j=A, B}\left[g_{j} a^{+}\left|0_{j}\right\rangle\left\langle e_{j}\left|+\Omega_{j}(t)\right| e_{j}\right\rangle\left\langle 1_{j}\right|+H . c .\right] \\
& -i \frac{\kappa}{2} a^{+} a-i \frac{\Gamma}{2} \sum_{j=A, B}\left|e_{j}\right\rangle\left\langle e_{j}\right|
\end{aligned}
$$

where $\kappa$ is the cavity decay rate, and $\Gamma$ is the spontaneous emission rate with respect to the excited state $|e\rangle$. We set the values of other experimental parameters as $g_{A}=$ $g_{B}=g_{0}=2 \pi \times 1 \mathrm{GHz}, \Omega_{m} \simeq 2 \pi \times 470 \mathrm{MHz}, \tau_{A}=6.8 \mathrm{~ns}$, $\tau_{B}=5 \mathrm{~ns}, \triangle \tau=1.8 \mathrm{~ns}$, and $\kappa=\Gamma=g_{0} / 10$, which fulfill the adiabatic conditions $\Omega_{m} \triangle \tau \gg 1$ and $g_{j} \triangle \tau \gg 1$ [46]. In our case, the time delay $\left(\tau_{A}-\tau_{B}\right)$ between two pulses $\Omega_{A}$ and $\Omega_{B}$ of the same step is chosen to be equal to $\triangle \tau$ to minimize the nonadiabatic losses [47]. Moreover, the condition $g_{j} \gg \Omega_{m}$ guarantees that the cavity mode is negligibly populated during the interaction with the pulses, as demonstrated in Fig. 5.
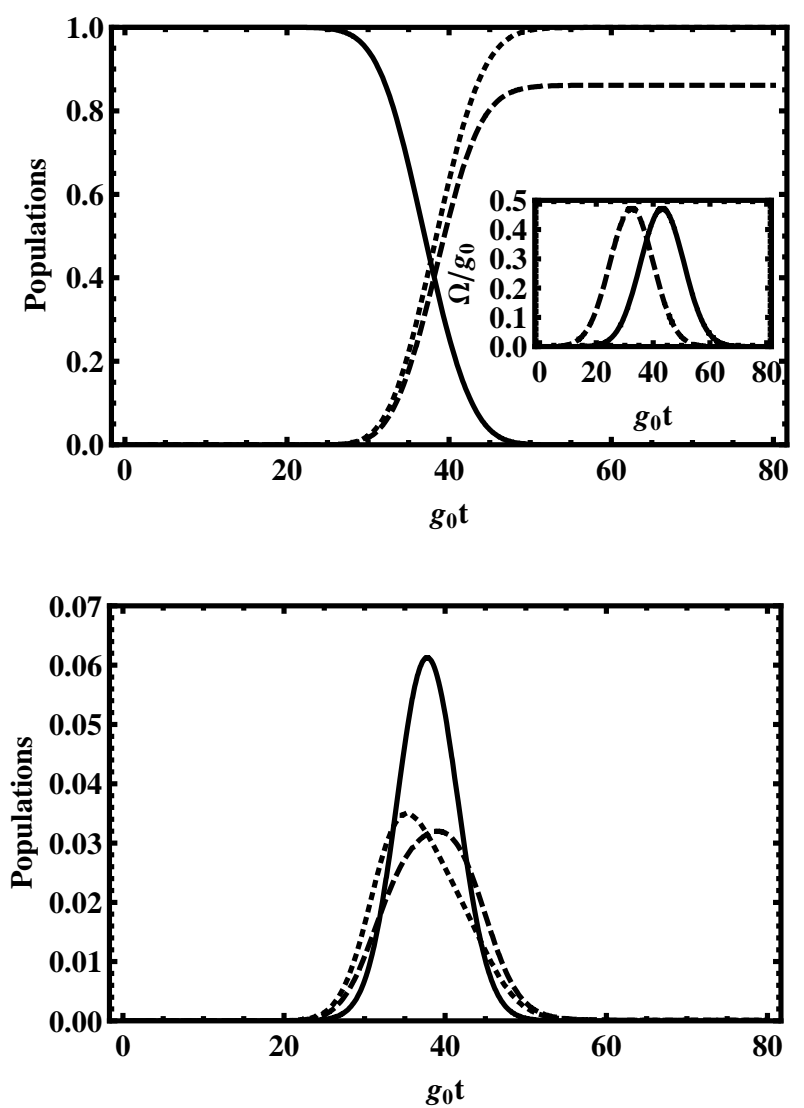

FIG. 5: Numerical simulation exhibiting the populations versus $g_{0} t$. Top: the solid curve denotes the population of $\left|1_{A}\right\rangle\left|0_{B}\right\rangle\left|0_{c}\right\rangle$. The dotted and dashed curves represent the population of $\left|0_{A}\right\rangle\left|1_{B}\right\rangle\left|0_{C}\right\rangle$ in the ideal and decay cases, respectively. The inset shows the Rabi frequencies $\Omega_{A}$ (solid line) and $\Omega_{B}$ (dashed line). Bottom: the solid, dotted, and dashed curves represent the populations of $\left|0_{A}\right\rangle\left|0_{B}\right\rangle\left|1_{C}\right\rangle$, $\left|e_{A}\right\rangle\left|0_{B}\right\rangle\left|0_{C}\right\rangle$, and $\left|0_{A}\right\rangle\left|e_{B}\right\rangle\left|0_{c}\right\rangle$, respectively, where the parameters used are $\Omega_{m} \triangle \tau=5$ and $g_{0} \triangle \tau=11$.

We emphasize that adiabatic passage method could make the QIT process robust against the fluctuations of experimental parameters, such as small variations of the peak Rabi frequencies $\Omega_{m}$, and of the WGM-NV coupling strength $g_{0}$. Once the adiabatic conditions $\Omega_{m} \triangle \tau \gg$ $1, g_{j} \triangle \tau \gg 1$ and $g_{j} \gg \Omega_{m}$ could be well satisfied, our scheme neither requires accurate manipulation of the intensities of the laser pulses, nor demands precise control of the WGM-NV interaction. The only thing we need to do is keeping the phase differences stable between the laser pulses $\Omega_{A}(t)$ and $\Omega_{B}(t)$, which is easily controllable experimentally. It should be mentioned that the fidelity 
of the Bell state $|B e l l\rangle_{A B}$ depends only on the ratio of the WGM-NV coupling constants $g_{A}$ and $g_{B}$ after the laser pulses $\Omega_{A}(t)$ and $\Omega_{B}(t)$ are turned off. If $g_{A}$ and $g_{B}$ are not the same, the two NV centers will be prepared in the state $\left(g_{A}\left|1_{A}\right\rangle\left|0_{B}\right\rangle+g_{B}\left|0_{A}\right\rangle\left|1_{B}\right\rangle\right) / \sqrt{g_{A}^{2}+g_{B}^{2}}$ with the fidelity $F=\left(g_{A}+g_{B}\right)^{2} /\left[2\left(g_{A}^{2}+g_{B}^{2}\right)\right]$.

\section{FEASIBILITY AND CHALLENGE}

We examine the feasibility of our scheme and survey the relevant experimental parameters. As stated above, NV centers are well suited for cavity QED because they have a long electron spin relaxation time and their electronic states can be initialized, manipulated, and measured through highly stable optical and microwave excitations at room temperature. Additionally, WGM microcavities exhibit exciting characteristics such as extremely high-quality factor $Q$, small mode volume $V_{m}$, and excellent scalability, which makes it possible to achieve high concentration of the optical field and relatively long photon confinement times. Experimentally, single N-V centers strongly coupling to a WGM have been demonstrated in different kinds of microcavities [21 23], which are great advances in WGM-based cavity QED research.

In the composite nanocrystal-microsphere system, we focus our attention on the dipole transition $|0\rangle \leftrightarrow|e\rangle$ with a ZPL at $\lambda=637 \mathrm{~nm}$ of the NV centers in diamond nanocrystal. In above-mentioned WGM-NV experiments, the NV centers actually interact with the evanescent field of the WGM. The evanescent field is of importance because it offers the effective way to energy exchange between the WGM and the external NV center. Thus, we assume that single NV centers are located near the microcavity surface in order for the WGM-NV coupling $g_{\max }$ to reach $2 \pi \times 1 \mathrm{GHz}[20$ ] with the parameters in the Sec. 2.2. In the fused-silica microsphere cavity, the small radius of $10 \mu \mathrm{m}$ could lead to a vacuum electric field of $150 \mathrm{~V} / \mathrm{cm}$ at the sphere surface and to the $Q$ factor exceeding $10^{9}$, which imply the WGM decay rate to be $\kappa=\omega_{e 0} / Q=2 \pi \times 0.47 \mathrm{MHz}$. Experimental studies have demonstrated $Q$ factors approaching $10^{10}$ in a silica WGM microsphere [35, 48], with values exceeding $10^{8}$ readily achievable over a broad range of cavity diameters and wavelengths. The strong coupling strongly depends on the critical photon number $n_{0}=\Gamma_{0}^{2} /\left(2 g_{\max }^{2}\right)$ and the critical atom number $N_{0}=2 \Gamma_{0} \kappa /\left(g_{\max }^{2}\right)$, which gives the number of photons required to saturate a NV-qubit, and the number of NV-qubit required to have an appreciable effect on the WGM cavity transmission, respectively [20]. Based on these parameters, one can find that the strong coupling conditions $g_{\max } \gg \kappa, \Gamma_{0}$ and $\left(n_{0}, N_{0}\right) \ll 1$ could be well satisfied in our scheme.

Nevertheless, in realistic experiments, WGMs are dominantly confined inside of the microcavity body, and only the remaining energy of the WGM can be resident in the exterior evanescent field. Thus the coherent coupling strength cannot reach its maximum. This is the reason that the maximal WGM-NV coupling in current experiments only reaches $2 \pi \times 300 \mathrm{MHz}$ [23]. There is an increasing interest in the development of alternative microcavity systems. We note that a recent experiment [49] has demonstrated an enhanced coherent interaction between the WGM and quantum dots using a kind of plasmonic WGM highly localized on the exterior surface of a metal-coated microtoroidal. Another alternative soultion is using the silica microtoroidal coated with a highrefractive-index (HRI) nanolayer [50]. The key idea is that this HRI nanolayer can compress the radial WGM field and move the WGM field in the coated microtoroidal to the outside of the silica surface.

Note that the preparation of entangled states and implementation of QIT via dark states are similar in spirit to the other atom [43] and atom-like systems [51]. However, we consider here a different system, and our proposal has several merits as follows. Firstly, the NV center in diamond is the only currently known viable solid-state qubit at room-temperature, and the center's highly localized bound states are well isolated from sources of decoherence. So the ground state can exhibit extremely long spin coherence time of up to millisecond, which is close to the regime needed for quantum error correction 52]. Secondly, the proposed QIT protocol has the potential of scalability because our QIT protocol does not require identical WGM-NV coupling strength, which implies that neither identical qubits nor exact placement of NV centers in cavities is needed. Thirdly, as the NV center nanocrystals are required to be attached along the equator of the microsphere with spacing bigger than the laser wavelength, individual addressing is not an obstacle in our scheme.

The two schemes above require different conditions in implementation. For example, in the first scheme the WGMs are detuned from the transitions in the NV centers, while the second scheme requires resonant coupling between the WGMs and the NV centers. If we employ the same WGM cavities to accomplish the schemes, the detuned and the resonant couplings regarding the WGM radiation could be achieved by changing the temperature of the system [21].

In current experiments, the electron spin relaxation time $T_{1}$ of diamond $\mathrm{NV}$ centers ranges from $6 \mathrm{~ms}$ at room temperature [11] to sec at low temperature. In addition, the dephasing time $T_{2}=350 \mu$ s induced by the nearby nuclear-spin fluctuation has been reported [53]. A latest experimental progress [54] with isotopically pure diamond sample has demonstrated a longer $\mathrm{T}_{2}$, i.e., $\mathrm{T}_{2}=2$ ms. In our scheme, the operation times for preparing four-qubit $\mathrm{W}$ state, the two-qubit Bell state, and for accomplishing QIT are about $31.3 \mathrm{~ns}, 6.786 \mathrm{~ns}$, and 8.0 ns, respectively. Hence even at room temperature, up to $10^{3} \sim 10^{4}$ gate operations are feasible under the present experimental conditions.

For more technical aspects, to make sure the NV centers to be strongly and nearly equally coupled to the WGM cavity, we have to simultaneously attach sepa- 
rate NV centers around the equator of a single fusedsilica microsphere resonator. The experimental difficulty lies in how to fix the nanocrystals appropriately with respect to the WGM. So far the strong coupling between a single NV center and the WGM of a silica microsphere [21] and a gallium-phosphide microdisk [23], and between two NV centers and a polystyrene microsphere [22] have been experimentally achieved. So we wish this would be soon extended to more NV centers. Further consideration would involve nuclear spins in the NV centers, which are more suitable to store quantum information due to longer decoherence time. In this sense, we may also consider quantum computation with NV centers combining nuclear spins with electron spins, i.e., encoding the qubits in the nuclear spins and employing the electron spins as ancillas. The hyperfine interaction helps to transfer the generated entanglement of the electron spins to the corresponding nuclear spins of NV centers [7].

\section{CONCLUSION AND ACKNOWLEDGEMENTS}

In conclusion, we have proposed two schemes to prepare W state and Bell state with separate NV centers in the diamond nanocrystal-microsphere system, respectively. In both of these schemes, the cavity decay and the spontaneous emission from the excited states could be effectively suppressed. Particularly, in the latter scheme with the adiabatic passage, the QIT is robust against the experimental parameter fluctuation.

Our ideas could be applied to other cavity systems, besides the WGM-type cavities. The number of the entangled NV centers depends on the ratio of the size of the employed microcavity to the nanocrystal size. For more NV centers to be entangled, detection of emitted photons by parity projection would be necessary [14]. In this sense, we argue that our present work has demonstrated a building block for a large-scaled NV center system, which would be feasible in the near future.

WLY thanks Zhang-qi Yin for enlightening discussions. This work is supported by National Natural Science Foundation of China under No. 10974225, by Chinese Academy of Sciences, and by 973 project.
[1] Gruber A, Dräbenstedt A, Tietz C, Fleury L, Wrachtrup J and Borczyskowski C 1997 Science 2762012

[2] Childress L, Gurudev Dutt M V, Taylor J M, Zibrov A S, Jelezko F, Wrachtrup J, Hemmer P R and Lukin M D 2006 Science $\mathbf{3 1 4} 281$

[3] Togan T, Chu Y, Trifonov A S, Jiang L, Maze J, Childress L, Dutt M V G, Srensen A S, Hemmer P R, Zibrov A S and Lukin M D 2010 Nature 466730

[4] Stoneham A M 2009 Physics 234

[5] Shi F, Rong X, Xu N, Wang Y, Wu J, Chong B, Peng X, Kniepert J, Schoenfeld R -S, Harneit W, Feng M and Du J -F 2010 Phys. Rev. Lett. 105040504

[6] Jelezko F, Gaebel T, Popa I, Gruber A and Wrachtrup J 2004 Phys. Rev. Lett. 92076401

[7] Gurudev Dutt M V, Childress L, Jiang L, Togan E, Maze J, Jelezko F, Zibrov A S, Hemmer P R and Lukin M D 2007 Science 3161312

[8] Hanson R, Mendoza F M, Epstein R J and Awschalom D D 2006 Phys. Rev. Lett. 97087601

[9] Jacques V, Neumann P, Beck J, Markham M, Twitchen D, Meijer J, Kaiser F, Balasubramanian G, Jelezko F and Wrachtrup J 2009 Phys. Rev. Lett. 102057403

[10] Jiang L, Hodges J S, Maze J R, Maurer P, Taylor J M, Cory D G, Hemmer P R, Walsworth R L, Yacoby A, Zibrov A S and Lukin M D 2009 Science 326267

[11] Neumman P, Mizuochi N, Rempp F, Hemmer P, Watanabe H, Yamasaki S, Jacques V, Gaebel T, Jelezko F and Wrachtrup J 2008 Science 3201326

[12] Cappellaro P, Jiang L, Hodges J S and Lukin M D 2009 Phys. Rev. Lett. 102210502

[13] Neumman P, Kolesov R, Naydenov B, Beck J, Rempp F, Steiner M, Jacques V, Balasubramanian G, Markham
M L, Twitchen D J, Pezzagna S, Meijer J, Twamley J, Jelezko F and Wrachtrup J 2010 Nat. Phys. 6249

[14] Benjamin S C, Lovett B W and Smith J M 2009 Laser \& Photo. Rev. 3556

[15] Yang W L, Yin Z Q, Xu Z Y, Feng M and Du J F 2010 Appl. Phys. Lett. 96241113

[16] Acosta V M, Bauch E, Ledbetter M P, Santori C, Fu K M C, Barclay P E, Beausoleil R G, Linget H, Roch J F, Treussart F, Chemerisov S, Gawlik W and Budker D 2009 Phys. Rev. B 80115202

[17] Manson N B, Harrison J P and Sellars M J 2006 Phys. Rev. B $\mathbf{7 4} 104303$

Rogers L J, Armstrong S, Sellars M J and Manson N B 2008 New. J. Phys. 10103024

[18] Vahala K J 2003 Nature 424839

[19] Buck J R and Kimble H J 2003 Phys. Rev. A 67033806

[20] Spillane S M, Kippenberg T J, Vahala K J, Goh K W, Wilcut E and Kimble H J 2005 Phys. Rev. A 71013817

[21] Park Y-S, Cook A K and Wang H 2006 Nano Lett. 62075

[22] Schietinger S, Schröder T and Benson O 2008 Nano Lett. 83911

[23] Barclay P E, Fu K-M C, Santori C and Beausoleil R G 2009 Appl. Phys. Lett. 95191115

[24] Englund D, Shields B, Rivoire K, Hatami F, Vuckovic J, Park H and Lukin M D 2010 arXiv:1005.2204

[25] Armani D K, Kippenberg T J, Spillane S M and Vahala K J 2003 Nature 421925

McRae T-G and Browen W P 2009 Phys. Rev. A 80 010303(R)

Yin Z-Q and Han Y-J 2009 Phys. Rev. A 79024301

Strekalov D V, Schwefel H G L, Savchenkov A A, Matsko A B, Wang L J and Yu N 2009 Phys. Rev. A 80033810 
[26] Yang Y-D, Huang Y-Z and Chen Q 2007 Phys. Rev. A $\mathbf{7 5} 013817$

[27] Min B, Ostby E, Sorger V, Ulin-Avila E, Yang L, Zhang $\mathrm{X}$ and Vahala K 2009 Nature 457455 Srinivasan K and Painter O 2007 Phys. Rev. A 75023814

[28] Cai M, Painter O and Vahala K J 2000 Phys. Rev. Lett. 8574

Barker P F 2010 Phys. Rev. Lett. 105073002

[29] Dür W, Vidal G and Cirac J I 2000 Phys. Rev. A 62 062314

Yu C-S, Yi X X, Song H-S and Mei D 2007 Phys. Rev. A 75044301

Guo G-P, Li C-F, Li J and Guo G-C 2002 Phys. Rev. A 65042102

[30] Bergmann K, Theuer H and Shore B W 1998 Rev. Mod. Phys. 701003

Kis Z, Karpati A, Shore B W and Vitanov N V 2004 Phys. Rev. A 70053405

[31] Yang C-P, Chu S-I and Han S 2004 Phys. Rev. Lett. 92 117902

[32] Spillane S M, Kippenberg T J, Painter O J and Vahala K J 2003 Phys. Rev. Lett. 91043902

[33] Santori C, Tamarat P, Neumann P, Wrachtrup J, Fattal D, Beausoleil R G, Rabeau J, Olivero P, Greentree A D, Prawer S, Jelezko F and Hemmer P 2006 Phys. Rev. Lett. 97247401

[34] Su C-H, Greentree A D and Hollenberg L C L 2008 Optics Express 166240

[35] Gorodetsky M L, Savchenkov A A and Ilchenko V S 1996 Opt. Lett. 21453

[36] Tamarat P, Manson N B, Harrison J P, McMurtrie R L, Nizovtsev A, Santori C, Beausoleil R G, Neumann P, Gaebel T, Jelezko F, Hemmer P and Wrachtrup J 2008 New. J. Phys. 10045004

[37] Zheng S B and Guo G C 2001 Phys. Rev. A 63044302 Feng M 2002 Phys. Rev. A 66054303

[38] Wu C, Feng X-L, Yi X X, Chen I M and Oh C H 2008 Phys. Rev. A $\mathbf{7 8} 062321$

[39] Neumann P, Kolesov R, Jacques V, Beck J, Tisler J, Batalov A, Rogers L, Manson N B, Balasubramanian G, Jelezko F and Wrachtrup J 2009 New. J. Phys. 11013017

[40] Clark S G and Parkins A S 2003 Phys. Rev. Lett. 90 047905

Pellizzari T 1997 Phys. Rev. Lett. 795242
Yin Z-Q, Li F-L and Peng P 2007 Phys. Rev. A 76062311

[41] The spontaneous decay rates from $|e\rangle$ to $|1\rangle$ and from $|e\rangle$ to $|0\rangle$ are assumed to be equal.

[42] Walls D F and Milburn G J 1994 Quantum Optics Springer-Verlag, Berlin.

[43] Parkins A S, Marte P, Zoller P and Kimble H J 1993 Phys. Rev. Lett. 713095

Pellizzari T, Gardiner S A, Cirac J I and Zoller P 1995 Phys. Rev. Lett. $\mathbf{7 5} 3788$

[44] Bergmann K, Theuer H and Shore B W 1998 Rev. Mod. Phys. 701003

Vitanov N V, Fleischhauer M, Shore B W and Bergmann K 2001 Adv. At., Mol., Opt. Phys. 4655

[45] The WGM state could be detected by dint of an auxiliary NV center initially in the state $|0\rangle$. The transition $|0\rangle \Longleftrightarrow|e\rangle$ is resonant with the WGM for an interaction time $t=\pi / 2 g$ with $g$ the coupling strength due to the WGM. If the auxiliary NV center is detected in the state $|0\rangle$, the state of WGM was initially in the $\left|0_{c}\right\rangle$ state.

[46] Vitanov N V, Suominen K-A and Shore B W $1999 \mathrm{~J}$. Phys. B: Mol. Opt. Phys. 324535

[47] Sangouard N, Lacour X, Guérin S and Jauslin H R 2005 Phys. Rev. A $\mathbf{7 2} 062309$

[48] Vernooy D W, Ilchenko V S, Mabuchi H, Streed E W and Kimble H J 1998 Opt. Lett. 23247

[49] Xiao Y-F, Zou C-L, Li B-B, Li Y, Dong C-H, Han Z-F and Gong Q 2010 Phys. Rev. Lett. 105153902

[50] Xiao Y-F, Zou C-L, Xue P, Xiao L, Li Y, Dong C-H, Han Z-F and Gong Q 2010 Phys. Rev. A 81053807

[51] Brun T A and Wang H 2000 Phys. Rev. A 61032307

[52] Wrachtrup J 2010 Proc Natl Acad Sci USA 1079479

Weber J R, Koehl W F, Varley J B, Janotti A, Buckley B B, Walle C G V and Awschalom D D 2010 Proc Natl Acad Sci USA 1078515

[53] Gaebel T, Domhan M, Popa I, Wittmann C, Neumann P, Jelezko F, Rabeau J R, Stavrias N, Greentree A D, Prawer S, Meijer J, Twamley J, Hemmer P R and Wrachtrup J 2006 Nat. Phys. 2408

[54] Balasubramanian G, Neumann P, Twitchen D, Markham M, Kolesov R, Mizuochi N, Isoya J, Achard J, Beck J, Tissler J, Jacques V, Hemmer P R, Jelezko F and Wrachtrup J 2009 Nat. Mater. 8383 